\title{
Anharmonic effects on the structural and vibrational properties of the ethyl radical: A path integral Monte Carlo study
}

\author{
Telesforo López-Ciudad and Rafael Ramírez ${ }^{\text {a) }}$ \\ Instituto de Ciencia de Materiales, Consejo Superior de Investigaciones Científicas (C.S.I.C.), \\ Cantoblanco, 28049 Madrid, Spain \\ Joachim Schulte \\ Bruker Biospin GmbH, Silberstreifen, D-76287 Rheinstetten, Germany \\ Michael C. Böhm \\ Eduard-Zintl-Institut für Anorganische und Physikalische Chemie, Physikalische Chemie III, \\ Technische Universität Darmstadt, Petersenstrasse 20, D-64287 Darmstadt, Germany
}

(Received 19 March 2003; accepted 21 May 2003)

\begin{abstract}
The structural and vibrational properties of the ethyl radical have been investigated by a series of finite temperature simulations that treat the nuclei as quantum particles. The potential energy surface of the electronic ground state has been described by a nonorthogonal tight-binding Hamiltonian that provides results in reasonable agreement with ab initio methods. The quantum nature of the nuclei has been described by path integral Monte Carlo simulations at temperatures between 25 and 1000 K. Special interest deserves the determination of anharmonic and tunneling effects in the zero-point vibrational structure. In particular, we have studied the influence of anharmonic effects both on the mean value and the quantum fluctuations of equilibrium bond lengths and bond angles. The local structure of the radical center is found to be planar as a result of the zero-point motion of the atomic nuclei, even though the minimum energy configuration exhibits a pyramidal structure for this center. Anharmonic effects in the fundamental vibrational modes of the molecule are studied by a nonperturbative approach based on the centroid density. This function is a path integral concept that provides information on the static response of the system to applied external forces. Our study reveals a softening of the stretching modes associated with the $\mathrm{C}-\mathrm{H}$ bonds and a hardening of the out-of-plane rocking motion of the methylene group. Both effects are in good agreement with experimental and $a b$ initio data. The softening of the $\mathrm{C}-\mathrm{C}$ stretching mode predicted by our simulations suggests a revision of the currently accepted experimental assignment for two fundamental vibrations of the ethyl radical. The tunneling of an $\mathrm{H}$ atom between the methyl and methylene groups has been investigated. These simulations should contribute to the open question whether or not this process is responsible for the changes in the electron spin resonance spectrum at low temperatures. (C) 2003 American Institute of Physics. [DOI: 10.1063/1.1591731]
\end{abstract}

\section{INTRODUCTION}

Hydrocarbon radicals are unstable chemical species that play an important role in many chemical processes, as in hydrocarbon combustion chemistry, in organic reactions involving halogen-substituted hydrocarbons, and in addition reactions to a carbon-carbon double bond. The simplest of these addition reactions is the combination of a hydrogen atom with ethylene to form the ethyl radical, $\mathrm{C}_{2} \mathrm{H}_{5}$. In contrast to the methyl radical, the structure of the ethyl and all larger alkyl radicals is poorly characterized. The presence of large amplitude skeletal and torsional motions is expected to determine both the structure and dynamics of these unstable species. $^{1}$

A large number of experimental investigations have focused on spectroscopic properties of the ethyl radical, such as vibrational frequencies, the internal rotation dynamics, hyperfine constants, and electronic transitions. Spectroscopic

${ }^{\text {a)} E l e c t r o n i c ~ m a i l: ~ r a m i r e z @ i c m m . c s i c . e s ~}$ studies of unstable species have been often possible by matrix isolation techniques. Here the unstable species can become long-lived, as they remain isolated in nonreactive criocrystals such as solid parahydrogen or argon. ${ }^{2}$ The IR spectra of the ethyl radical was observed in argon matrices at $10 \mathrm{~K}$ by Pacansky et al. ${ }^{3,4}$ The spectra of isotopic species in combination with $a b$ initio calculations provided the basis for the assignment of 10 out of the 15 fundamental vibrational modes of the molecule. A more recent IR study of the $\mathrm{C}_{2} \mathrm{H}_{5}$ radical in an argon matrix has led to the observation of two additional bands. ${ }^{5}$ The small differences between the experimental data of Refs. 3-5 were attributed to the perturbing effect of the different matrix environments on the vibrational frequencies. An IR study of the radical in a matrix of solid parahydrogen at $8 \mathrm{~K}$ provided evidence for the three highest vibrational modes of the molecule, but failed to detect the corresponding peaks for the remaining modes. ${ }^{6}$

The two lowest-frequency vibrational modes of ethyl are the torsion and the out-of-plane rocking motion of the $\mathrm{CH}_{2}$ group. Both modes are strongly coupled and act as active 
modes for the internal rotation of the molecule. The pure rotational spectrum of the torsional band has not been measured so far. However, a high resolution spectrum of the $\mathrm{CH}_{2}$ rocking fundamental at about $530 \mathrm{~cm}^{-1}$ has been analyzed at room temperature in the gas phase by IR laser transient absorption spectroscopy. The spectrum contains many torsionrotational transitions that provide evidence for a very low torsional barrier of about $17 \mathrm{~cm}^{-1}$ in the vibrational zeropoint configuration. ${ }^{1,7}$ High resolution IR spectra for both symmetric and asymmetric $\mathrm{CH}_{2}$ stretches, at about 3037 and $3129 \mathrm{~cm}^{-1}$, have been measured at $14 \mathrm{~K}$ in jet-cooled ethyl radicals. The analysis of the rotational structure of the vibrational bands leads to rotational constants that imply a $\mathrm{C}-\mathrm{C}$ bond distance of $149 \mathrm{pm}^{8}$ Experimental information on other bond distances or bond angles of ethyl has not been presented so far.

The presence of an unpaired electron in the ethyl radical has motivated a number of interesting investigations. The high-resolution electron spin resonance (ESR) spectrum of the ethyl radical in solid parahydrogen has been measured in a temperature range between 3.1 and $6.7 \mathrm{~K}$. The data rendered possible the determination of rotational level splittings in reasonable agreement with the results of IR absorption spectroscopy. ${ }^{2}$ The ESR spectrum of the ethyl radical obtained by the reaction of hydrogen atoms in an ethane matrix with a high degree of crystalline order shows changes in the widths and intensities of some individual lines upon cooling from 30 to $10 \mathrm{~K}$. These changes were tentatively attributed to the influence of internal tunneling rotations on the methylproton hyperfine splittings. ${ }^{9}$ However, a sound explanation of this experiment is still missing. Muon spin resonance experiments have been used to determine hyperfine parameters for the gas phase radical. ${ }^{10,11}$ Other ESR studies have led to hyperfine parameters in solid inert matrices. ${ }^{12-14}$ Electronic properties of the excited states of the ethyl radical have been investigated by photoionization mass spectrometry $^{15}$ and photoelectron spectroscopy. ${ }^{16}$

Theoretical studies including electronic correlation at different levels of sophistication have provided $a b$ initio geometries, ${ }^{15,17-21}$ harmonic vibrational wave numbers, ${ }^{15,17,18,22}$ and hyperfine constants ${ }^{23-27}$ for the ethyl radical. The calculation of the torsional potential associated with the internal rotation shows that the coupling between the internal rotation and the other normal modes of the molecule can have a substantial effect on the magnitude of the barrier itself. $^{28,29}$ The energy levels for the rotation and internalrotation of the ethyl radical have been studied by a model Hamiltonian fitted to reproduce experimental rotationaltorsional transitions. ${ }^{7}$ These data improve previous results obtained with a so-called rotation-contortion Hamiltonian. ${ }^{29}$ Calculated isotropic hyperfine coupling constants depend quite sensitively on the internal rotation as well as on the rocking motion of the methylene group. Therefore, an average over populated vibrational states is necessary to obtain good agreement to experiment. ${ }^{24,25}$ The theoretical treatment of vibrational effects also enables the study of isotopic substitution. The hyperfine coupling constants of the deuterium-, protium-, and muonium-substituted radicals have been calculated by a variation-perturbation approach. This method in- cludes only three normal modes of the radical in the vibrational average. ${ }^{26,27}$ The vibrational corrections for the coupling constants amount to $23 \%$ for the muoniumsubstituted radical and to 5\% for the protonated radical.

Previous theoretical investigations of the ethyl radical have either neglected anharmonic effects on the molecular properties, ${ }^{3,4,15,18,19,23,30}$ or have treated them either empirically, ${ }^{17,22}$ or by a vibrational average considering only some vibrational modes. ${ }^{24,27-29}$ The motivation of the present theoretical investigation is to present a more realistic treatment of anharmonic and tunneling effects on the structural and dynamic properties of the ethyl radical at finite temperatures. A prerequisite for this strategy is the employment of a technique that takes into account the quantum nature of the atomic nuclei. Furthermore, a realistic description of the Born-Oppenheimer potential energy surface (PES) for the electronic ground state of this molecule is also needed. In order to meet these two requisites, we have combined finite temperature Feynman path integral Monte Carlo (PI MC) simulations for the atomic nuclei ${ }^{31,32}$ with an electronic tightbinding (TB) Hamiltonian developed recently. The electronic formalism has been parametrized on the basis of density functional results. ${ }^{33,34}$ Then, our computational approach treats both the electrons and the nuclei of the ethyl radical as quantum particles. In the last years, the study of solids and molecules at finite temperatures by combining electronic structure methods and path integral simulations for the atomic nuclei has led to a number of stimulating contributions. ${ }^{35-41}$ Previously we have employed this approach to study ground and excited state properties of ethylene $^{42,43}$ as well as the effect of nuclear quantum fluctuations on calculated nuclear magnetic resonance shieldings. ${ }^{44,45}$

To assess the influence of the quantum fluctuations of the nuclei on the structural and vibrational properties of the ethyl radical, we have compared the results of the quantum PI MC simulations with results of classical MC simulations, where the atomic nuclei are treated as classical particles. In particular, we have studied anharmonic effects in geometrical parameters (bond distances and bond angles), in the local structure of the radical center (planar versus pyramidal configurations), and in the internal energy of the molecule as a function of temperature. Anharmonic shifts in the vibrational wave numbers of ethyl have been derived with the help of an approach based on the linear response of the molecule to small external forces. ${ }^{46}$ The coherent tunneling of an $\mathrm{H}$ atom has been studied as a possible mechanism to explain the changes in the ESR spectrum below $30 \mathrm{~K} .{ }^{9}$

This paper is organized as follows: Section II summarizes the computational method used in our simulations. The PES derived by the employed TB Hamiltonian is compared to available $a b$ initio data in Sec. III, which is divided into two subsections. In Sec. III A we analyze the geometry of two molecular configurations and in Sec. III B we consider the harmonic vibrational wave numbers. The results of the PI MC simulations are presented in Sec. IV, which includes several subsections. Section IV A focuses on the equilibrium molecular structure at $25 \mathrm{~K}$. Anharmonic effects on the fundamental vibrational wave numbers are studied in Sec. IV B. 
The temperature dependence of the internal energy is presented in Sec. IV C. The tunneling of an $\mathrm{H}$ atom between the methyl $\left(\mathrm{CH}_{3}\right)$ and methylene $\left(\mathrm{CH}_{2}\right)$ groups is studied in Sec. IV D. Concluding remarks are given in Sec. V.

\section{COMPUTATIONAL METHOD}

The validity of the Born-Oppenheimer approximation leading to the movement of the nuclei on a PES defined by the electronic energy is assumed in our simulations. The nonorthogonal TB model of Seifert et al. has been used to calculate the PES of the ethyl radical. ${ }^{33,34}$ This method leads to geometries, vibrational frequencies, and reaction energies of high accuracy. In particular, it has been proven to be sufficiently accurate and transferable for complex carbon and hydrocarbon systems. ${ }^{33,34}$ A critical review on the capability of tight-binding methods for the modeling of materials can be found elsewhere. ${ }^{47}$

In the path-integral formulation of statistical mechanics, the partition function of a quantum system is evaluated through a discretization of the density matrix along cyclic paths, composed of a finite number of steps $N .^{31,48-51}$ In the numerical simulations, this Trotter number $N$ causes the appearance of $N$ "replicas" of a given quantum particle. Thus, the practical implementation of the method relies on an isomorphism between the quantum system and a classical one, defined by replacing each quantum particle (here, each atomic nucleus of the ethyl radical) by a ring polymer of $N$ classical particles. They are connected by harmonic springs with a temperature-dependent force constant. ${ }^{49,50}$ In our $n$-body system, the set of Cartesian coordinates of the $n$ atomic nuclei is defined as a $3 n$-dimensional vector $\left(x_{1}, \ldots, x_{i}, \ldots, x_{3 n}\right)$, while the coordinates of the ring polymers are denoted as $x_{i p}$, where the index $p$ varies from 1 to $N$.

The sampling of the configuration space of the ring polymers has been carried out by the Metropolis Monte Carlo method at temperatures between 25 and $1000 \mathrm{~K}^{52}$ A Monte Carlo step (MCS) is defined by: (i) an attempt of a sequential movement of the $n \times N$ Cartesian coordinates, $x_{i p}$, that define the ring polymers, and (ii) an attempt of a sequential rigid movement of the $n$ ring polymers. For a given temperature a typical run consisted of the generation of $3 \times 10^{4} \mathrm{MCS}$ for system equilibration, followed by $2.7 \times 10^{5} \mathrm{MCS}$ for the calculation of average properties. The simulations have been based on the "primitive" form of PI MC. Furthermore, the "crude" energy estimator has been used. ${ }^{53}$ The number of "replicas" $N$ in the quantum paths was taken to be temperature dependent, according to the relation $N T=6000 \mathrm{~K}$. This condition has been proven to give sufficiently converged results for ethylene. ${ }^{54}$ Within the employed algorithm, the classical limit is easily obtained by setting the Trotter number as $N=1$. More details on the actual application of the method can be found in Ref. 54 .

The spatial probability density of the "replicas" that build the ring polymers is an important observable. It maps the spatial probability density of the atomic nuclei. ${ }^{49,50}$ The centroid coordinate, $X_{i}$, is defined as the center-of-mass of the ring polymer associated with a given nucleus

$$
X_{i}=N^{-1} \sum_{p=1}^{N} x_{i p} .
$$

The centroid density, i.e., the spatial probability density of the ring polymer centroids, also carries interesting physical information. ${ }^{55-58}$ The linear response of the quantum system to vanishingly small external forces is given by the massweighted static susceptibility tensor, $\chi^{T},{ }^{46}$

$$
\chi_{i j}^{T}=\beta\left(m_{i} m_{j}\right)^{1 / 2} \delta X_{i} X_{j},
$$

where $\beta$ reads $\left(k_{B} T\right)^{-1}$ with $T$ symbolizing the temperature and $k_{B}$ the Boltzmann constant. $m_{i}$ is the mass of the nucleus associated to the coordinate $i$, and $\delta X_{i} X_{j}$ is the covariance of the centroid coordinates $X_{i}$ and $X_{j}$. The knowledge of $\chi^{T}$ is of large utility. It allows one to derive the harmonic linear response (HLR) approximation to the vibrational frequencies, i.e., a method that is applicable even for highly anharmonic systems (e.g., a particle undergoing quantum tunneling). This approach represents a significant improvement with respect to the standard harmonic approximation for the treatment of a vibrational problem. ${ }^{46}$ Remember that all commercial ab initio programs are only equipped with vibrational techniques based on the harmonic approximation. The HLR formula reads

$$
\omega_{i, \mathrm{HLR}}=\left(\frac{1}{\Delta_{i}}\right)^{1 / 2},
$$

where the $\Delta_{i}$ are the diagonal elements of the matrix defined by diagonalization of $\chi^{T}$, and $\omega_{i, \mathrm{HLR}}$ are the HLR approximation to the fundamental vibrational wave numbers, $\omega_{i}$. This approximation will be applied in Sec. IV B to study anharmonic effects on the fundamental modes of the ethyl radical. In Sec. III, we assess the capability of the TB model to describe the PES of the electronic ground state of ethyl.

\section{BORN-OPPENHEIMER POTENTIAL ENERGY SURFACE}

In Fig. 1 we display the two molecular configurations (staggered and eclipsed) of the ethyl radical that are studied in this section. The point group symmetry for both configurations is $C_{s}$ with a symmetry plane going through the atoms labeled as $\mathrm{H} 1, \mathrm{C} 1$, and $\mathrm{C} 2$. In the following, the TB results for relative energies, geometrical parameters, and harmonic vibrational wave numbers of ethyl will be compared to available $a b$ initio Hartree-Fock calculations with different treatments of the electronic correlation (i.e., to the MP2/6-31G* results of Ref. 15 and to the QCISD/6-3111G** data of Ref. 17) as well as to density functional calculations (i.e., to the TZ2Pf/B3LYP results of Ref. 18).

\section{A. Molecular structure}

The absolute energy minimum of the PES determined by the TB Hamiltonian corresponds to the staggered ethyl configuration. This result agrees with the $a b$ initio calculations. ${ }^{15,17}$ Geometrical parameters (bond lengths and angles) of the staggered configuration as derived by the TB model are compared to ab initio results in Table. I. The $\mathrm{C}-\mathrm{C}$ bond distance is about $1 \%$ smaller than the average of the $a b$ 

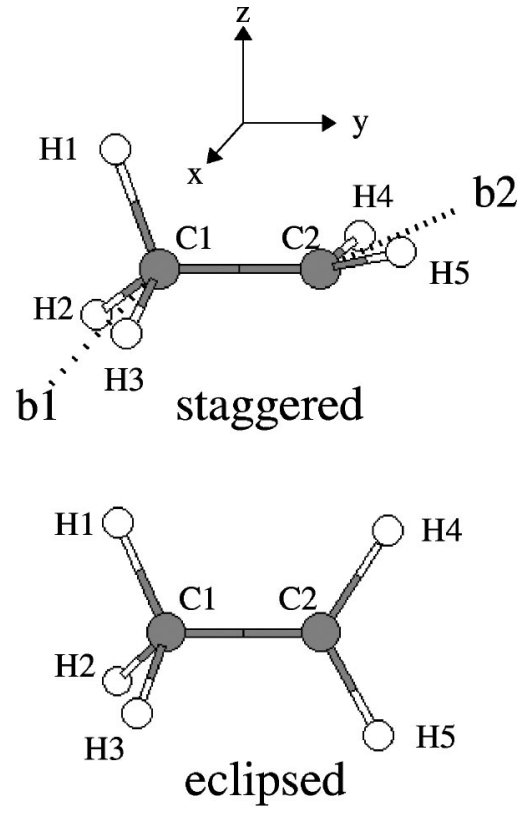

FIG. 1. Schematic representation of the staggered and eclipsed configurations of the ethyl radical. The numbering scheme of the atoms is given. The dotted lines labeled as $b_{1}$ and $b_{2}$ are the bisection of the angles $\mathrm{H} 2 \mathrm{C} 1 \mathrm{H} 3$ and $\mathrm{H} 4 \mathrm{C} 2 \mathrm{H} 5$, respectively. The orientation of the Cartesian axes is displayed at the top.

initio values, while the $\mathrm{C}-\mathrm{H}$ bond lengths are overestimated by about $3 \%$. These deviations are of the same order as those encountered in our TB study of ethylene, ${ }^{42}$ and point to a systematic error of the TB model. The ordering for the different $\mathrm{C}-\mathrm{H}$ bond lengths in the staggered structure, i.e., $d(\mathrm{C} 1-\mathrm{H} 1)>d(\mathrm{C} 1-\mathrm{H} 2)>d(\mathrm{C} 2-\mathrm{H} 4)$, is predicted correctly by the TB model. We note that the largest deviation found between the different ab initio data amounts to $1 \%$ for the $\mathrm{C}-\mathrm{C}$ bond and $0.5 \%$ for the $\mathrm{C}-\mathrm{H}$ distances.

The deviation found between the TB and ab initio values for some selected angles is typically smaller than $1 \%$. An exception is the rocking angle $\mathrm{C} 1-\mathrm{C} 2-\mathrm{b} 2$ (see Fig. 1), that shows a certain dispersion even within the ab initio calculations. This angle determines the local structure of the radical center at the $\mathrm{CH}_{2}$ group. A rocking angle of $180^{\circ}$ would

TABLE I. Geometrical parameters (bond lengths, $d$, and angles, $\alpha$ ) of the staggered minimum energy configuration of the ethyl radical. The parameters derived by the tight-binding method are compared to three sets of $a b$ initio values corresponding to the MP2/6-31G* calculation of Ref. 15, the QCISD/6-3111G** data of Ref. 17, and the TZ2Pf/B3LYP results of Ref. 18 , respectively. Bond lengths are given in $\mathrm{pm}$.

\begin{tabular}{ccccc}
\hline \hline$d, \alpha$ & TB & MP2 & QCISD & B3LYP \\
\hline C1-C2 & 146.6 & 148.9 & 149.9 & 148.4 \\
C1-H1 & 113.5 & 110.0 & 110.3 & 110.0 \\
C1-H2 & 112.2 & 109.3 & 109.6 & 109.2 \\
C2-H4 & 111.4 & 108.2 & 108.6 & 108.1 \\
C2-C1-H1 & $113.3^{\circ}$ & $111.9^{\circ}$ & $111.6^{\circ}$ & $111.9^{\circ}$ \\
$\mathrm{H} 2-\mathrm{C} 1-\mathrm{H} 3$ & $109.0^{\circ}$ & $108.1^{\circ}$ & $108.3^{\circ}$ & $108.2^{\circ}$ \\
$\mathrm{H} 4-\mathrm{C} 2-\mathrm{H} 5$ & $116.1^{\circ}$ & $117.2^{\circ}$ & $117.6^{\circ}$ & $117.6^{\circ}$ \\
$\mathrm{b} 1-\mathrm{C} 1-\mathrm{C} 2$ & $129.5^{\circ}$ & $128.4^{\circ}$ & $128.3^{\circ}$ & $129.5^{\circ}$ \\
$\mathrm{C} 1-\mathrm{C} 2-\mathrm{b} 2$ & $157.0^{\circ}$ & $168.3^{\circ}$ & $169.0^{\circ}$ & $173.8^{\circ}$ \\
\hline \hline
\end{tabular}

TABLE II. Geometrical parameters (bond lengths, $d$, and angles, $\alpha$ ) of the eclipsed structure of the ethyl radical. The parameters derived by the tightbinding method are compared to the ab initio MP2/6-31G* results of Ref. 15 . Bond lengths are given in $\mathrm{pm}$. The energetic separation from the staggered (minimum energy) configuration is given in $\mathrm{meV}$.

\begin{tabular}{ccc}
\hline \hline$d, \alpha$ & TB & MP2 \\
\hline $\mathrm{C} 1-\mathrm{C} 2$ & 146.4 & 148.9 \\
$\mathrm{C} 1-\mathrm{H} 1$ & 111.9 & 109.1 \\
$\mathrm{C} 1-\mathrm{H} 2$ & 113.1 & 109.7 \\
$\mathrm{C} 2-\mathrm{H} 4$ & 111.2 & 108.2 \\
$\mathrm{C} 2-\mathrm{H} 5$ & 111.2 & 108.1 \\
$\mathrm{C} 2-\mathrm{C} 1-\mathrm{H} 1$ & $111.4^{\circ}$ & $111.9^{\circ}$ \\
$\mathrm{C} 1-\mathrm{C} 2-\mathrm{H} 4$ & $121.3^{\circ}$ & $121.8^{\circ}$ \\
$\mathrm{C} 1-\mathrm{C} 2-\mathrm{H} 5$ & $120.9^{\circ}$ & $120.8^{\circ}$ \\
$\mathrm{H} 2-\mathrm{C} 1-\mathrm{H} 3$ & $105.2^{\circ}$ & $106.4^{\circ}$ \\
Energy difference & 8 & 6 \\
\hline \hline
\end{tabular}

imply a planar radical fragment. Both $a b$ initio and TB calculations predict a nonplanar radical unit. Note that the TB model yields an even larger deviation from planarity. However, we anticipate that zero-point effects are essential in the characterization of the local structure of this fragment.

The TB model predicts that the relative energy of the eclipsed configuration of ethyl is $8 \mathrm{meV}$ above the energy of the staggered one. This value is in reasonable agreement with the ab initio result of $6 \mathrm{meV} .{ }^{15}$ Geometrical parameters of the eclipsed configuration are summarized in Table. II. For this configuration only one set of $a b$ initio data is available. Deviations between the TB and ab initio results are of the same order as those found in the staggered configuration.

\section{B. Harmonic vibrational wave numbers}

The TB results for the wave numbers of the harmonic vibrational modes of the staggered ethyl configuration are compared in Table III to ab initio and experimental data. The type of the vibrational mode is given in the last column. We note that the TB result for most modes lies within the interval spanned by the lowest and the highest $a b$ initio wave number for the same mode. This can be considered as a satisfactory result. The wave numbers corresponding to the lowest vibrational modes, $\omega_{9}$ and $\omega_{15}$, are somewhat underestimated by the TB model. $\omega_{9}$ is associated to the rocking of the $\mathrm{CH}_{2}$ group, while $\omega_{15}$ corresponds to the torsion of this group. A schematic representation of both vibrational modes is given in Fig. 2. Although the dispersion of the TB and $a b$ initio harmonic wave numbers for the torsional mode is relatively large, the physical significance of this deviation should be considered as small. The reason is that the torsional mode is responsible for the internal rotation of the ethyl radical. The harmonic model, however, is not appropriate for the description of such a process.

The comparison between $a b$ initio harmonic wave numbers and experimental data is instructive in order to assess the importance of anharmonic effects in the vibrational modes. We have summarized in Table III the experimental results of Pacansky et al. ${ }^{3,4}$ (set A) and Chettur et al. (set B). ${ }^{5}$ We have employed an assignment for the experimental 
TABLE III. Wave numbers for the vibrational modes of the staggered structure of the ethyl radical. The harmonic wave numbers derived by the tight-binding method are compared to three sets of ab initio values corresponding to the MP2/6-31G* calculation of Ref. 15, the QCISD/6-3111G** data of Ref. 17, and the TZ2Pf/B3LYP results of Ref. 18, respectively. The two sets of experimental wave numbers were taken from Refs. 3 and 4 (set A), and from Ref. 5 (set B), respectively. The wave numbers are grouped according to the irreducible representations $\left(\Gamma_{i}\right)$ of the normal modes. A standard notation is employed for the type of vibrational modes: $\nu$ for stretch, $\delta$ for bend, and $\rho$ for rock. All units in $\mathrm{cm}^{-1}$.

\begin{tabular}{|c|c|c|c|c|c|c|c|c|}
\hline & $\Gamma_{i}$ & $\mathrm{~TB}$ & MP2 & QCISD & B3LYP & Expt. A & Expt. B & Type \\
\hline$\omega_{1}$ & $A^{\prime}$ & 3170 & 3244 & 3165 & 3142 & 3033 & 3036 & $\nu_{s} \mathrm{CH}_{2}$ \\
\hline$\omega_{2}$ & $A^{\prime}$ & 3102 & 3153 & 3082 & 3034 & 2920 & & $\nu_{a s} \mathrm{CH}_{3}$ \\
\hline$\omega_{3}$ & $A^{\prime}$ & 2934 & 3064 & 3033 & 2943 & 2842 & 2844 & $\nu_{s} \mathrm{CH}_{3}$ \\
\hline$\omega_{4}$ & $A^{\prime}$ & 1511 & 1553 & 1506 & 1481 & & & $\delta_{a s} \mathrm{CH}_{3}$ \\
\hline$\omega_{5}$ & $A^{\prime}$ & 1459 & 1538 & 1492 & 1466 & & 1383 & $\delta \mathrm{CH}_{2}$ \\
\hline$\omega_{6}$ & $A^{\prime}$ & 1411 & 1471 & 1425 & 1400 & 1366 & 1369 & $\delta_{s} \mathrm{CH}_{3}$ \\
\hline$\omega_{7}$ & $A^{\prime}$ & 1165 & 1110 & 1077 & 1060 & 1138 & 1133 & $\nu_{s} \mathrm{CC}$ \\
\hline$\omega_{8}$ & $A^{\prime}$ & 1015 & 1031 & 1002 & 980 & & 1025 & $\rho \mathrm{CH}_{3}$ \\
\hline$\omega_{9}$ & $A^{\prime}$ & 407 & 465 & 455 & 494 & 540 & 532 & $\mathrm{CH}_{2}$ rocking \\
\hline$\omega_{10}$ & $A^{\prime \prime}$ & 3284 & 3352 & 3268 & 3241 & 3112 & 3114 & $\nu_{a s} \mathrm{CH}_{2}$ \\
\hline$\omega_{11}$ & $A^{\prime \prime}$ & 3163 & 3199 & 3124 & 3078 & 2987 & 3036 & $\nu_{a s} \mathrm{CH}_{3}$ \\
\hline$\omega_{12}$ & $A^{\prime \prime}$ & 1484 & 1558 & 1508 & 1481 & 1440 & 1442 & $\delta_{a s} \mathrm{CH}_{3}$ \\
\hline$\omega_{13}$ & $A^{\prime \prime}$ & 1201 & 1249 & 1214 & 1194 & 1175 & 1185 & $\rho \mathrm{CH}_{3}$ \\
\hline$\omega_{14}$ & $A^{\prime \prime}$ & 759 & 841 & 815 & 813 & & & $\delta \mathrm{CH}_{2}$ \\
\hline$\omega_{15}$ & $A^{\prime \prime}$ & 48 & 163 & 150 & 96 & & & $\mathrm{CH}_{2}$ torsion \\
\hline
\end{tabular}

and calculated wave numbers that is currently accepted in the literature. ${ }^{3-5,18,22}$ Most $a b$ initio harmonic wave numbers are larger that the experimental ones, especially the wave numbers $\left(\omega_{1}, \omega_{2}, \omega_{3}, \omega_{10}\right.$, and $\left.\omega_{11}\right)$ associated with the stretching of the $\mathrm{C}-\mathrm{H}$ bonds. This expected systematic error of $a b$ initio harmonic wave numbers is often corrected by an empirical scaling factor smaller than one. ${ }^{17,22}$ However, an inverse anharmonic shift is found for the rocking mode, $\omega_{9}$, where the experimental wave number $\left(540 \mathrm{~cm}^{-1}\right.$, set A) is about $45-75 \mathrm{~cm}^{-1}$ larger than the $a b$ initio harmonic wave numbers. In the same line, we note that the experimental wave number for the $\mathrm{C}-\mathrm{C}$ bond stretch $\left(\omega_{7}=1138 \mathrm{~cm}^{-1}\right.$, set A) is about $25-80 \mathrm{~cm}^{-1}$ larger than the $a b$ initio values. It is of great interest to check whether or not the anharmonic effects derived from the TB model for the rocking $\left(\omega_{9}\right)$ and

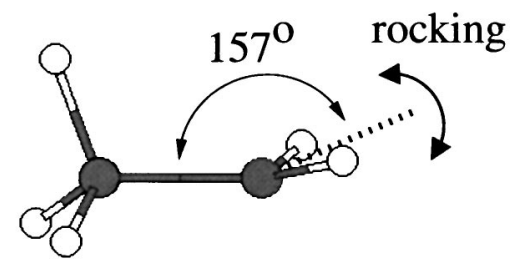

torsion

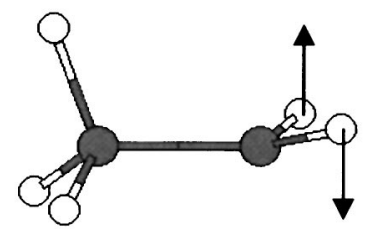

FIG. 2. Sketch of the atomic displacements corresponding to the rocking $\left(\omega_{9}\right)$ and torsional $\left(\omega_{15}\right)$ modes of the $\mathrm{CH}_{2}$ group of the ethyl radical. The rocking mode is associated with an oscillation of the rocking angle around its equilibrium value of $157^{\circ}$. The torsional mode implies a back and forth rotation of the methylene group with respect to the $\mathrm{C}-\mathrm{C}$ axis. the $\mathrm{C}-\mathrm{C}$ stretching $\left(\omega_{7}\right)$ modes lead to inverse anharmonic shifts. In other words, if it is possible that anharmonic corrections cause a hardening of the corresponding mode.

\section{PI MC SIMULATIONS}

The results of the PI MC simulations of ethyl are analyzed in the following subsections. Special interest deserves the identification of anharmonic affects in the structural and dynamic properties of ethyl. Our next topic is the study of the molecular structure at $T=25 \mathrm{~K}$. This temperature is low enough so that the molecule can be considered in its vibrational ground state for all modes, except for the torsional mode that is responsible of the internal rotation of the molecule.

\section{A. Molecular structure}

The probability density function (pdf) of the $\mathrm{C}_{2} \mathrm{H}_{5}$ nuclei at $25 \mathrm{~K}$ is displayed in Fig. 3 by a two-dimensional projection onto the $y z$ plane. The results of a classical MC simulation are shown in the top diagram, while those corresponding to the quantum PI MC simulation are displayed in the bottom diagram. The quantum fluctuations of the atomic nuclei are clearly seen by the larger spread of the pdfs in the PI MC simulation. The hydrogen atoms of the $\mathrm{CH}_{2}$ group spread over a large area due to the internal rotation of the molecule. We note that the internal rotation is active in both classical and quantum simulations at $25 \mathrm{~K}$. The internal rotation is more clearly seen in Fig. 4 by projections onto the $x z$ plane.

In Table IV we have summarized some equilibrium geometrical parameters of $\mathrm{C}_{2} \mathrm{H}_{5}$. The internal rotation causes the $\mathrm{C}-\mathrm{H}$ bond lengths of the methyl group to become identical, i.e., the three $\mathrm{H}$ atoms are dynamically equivalent. We recall that in the minimum energy configuration of ethyl, the three $\mathrm{H}$ atoms of the methyl group are topologically different (see Table I). The average bond lengths derived by quantum 

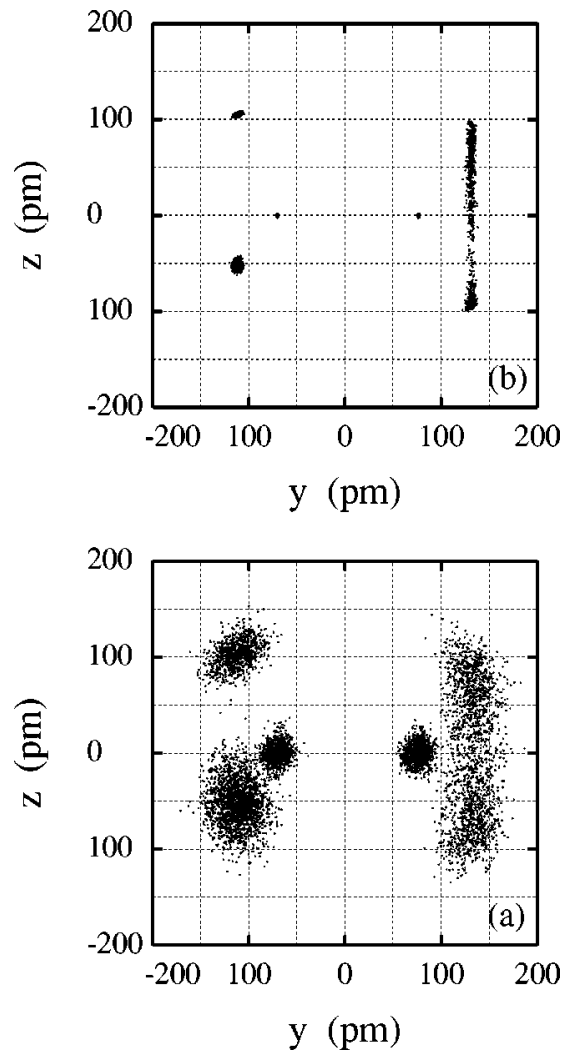

FIG. 3. Probability density function of the $\mathrm{C}_{2} \mathrm{H}_{5}$ nuclei projected onto the $y z$ plane as derived by quantum (a) and classical (b) simulations at $T$ $=25 \mathrm{~K}$.

and classical simulations are expected to be equal in the harmonic approximation. Therefore, the difference in the average bond lengths, $\langle d\rangle$, found in both simulations allows us to quantify the anharmonic effect due to the zero-point motion of the atomic nuclei. This effect causes an increase in the equilibrium $\mathrm{C}-\mathrm{C}$ bond length of $0.4 \%$ and an increase in the equilibrium $\mathrm{C}-\mathrm{H}$ bond lengths of about $2 \%$. The equilibrium $\mathrm{C}-\mathrm{C}$ bond length of $147.2 \mathrm{pm}$ is found to be in reasonable agreement with the value of $149 \mathrm{pm}$, which was derived from high resolution IR spectra. ${ }^{8}$

We note that, at $T=25 \mathrm{~K}$, the standard deviations, $\delta d$, of the bond length distributions are larger in the PI MC simulation than in the classical one. The larger fluctuations are a consequence of the zero-point motion of the atomic nuclei. For the $\mathrm{C}-\mathrm{H}$ bond distributions, the standard deviation derived by PI MC amounts to about $7 \%$ of the average bond length. For the $\mathrm{C}-\mathrm{C}$ bond distributions, $\delta d$ amounts to about $3 \%$ of the equilibrium bond length.

The average bond angles, $\langle\alpha\rangle$, in the quantum and classical simulations are only slightly different. Thus, one might infer that anharmonic effects in the bond angles are rather small. This conclusion is correct except for the rocking angle $\mathrm{C} 1-\mathrm{C} 2-\mathrm{b} 2$, where there appear important anharmonic effects in both quantum and classical simulations. A first indication of this behavior is provided by the unusually large value of the standard deviation, $\delta \alpha$, found for this angle in the classical simulation. As the rocking angle determines the local geometry of the radical center, it is interesting to study the probability density function of the pyramidalization co-
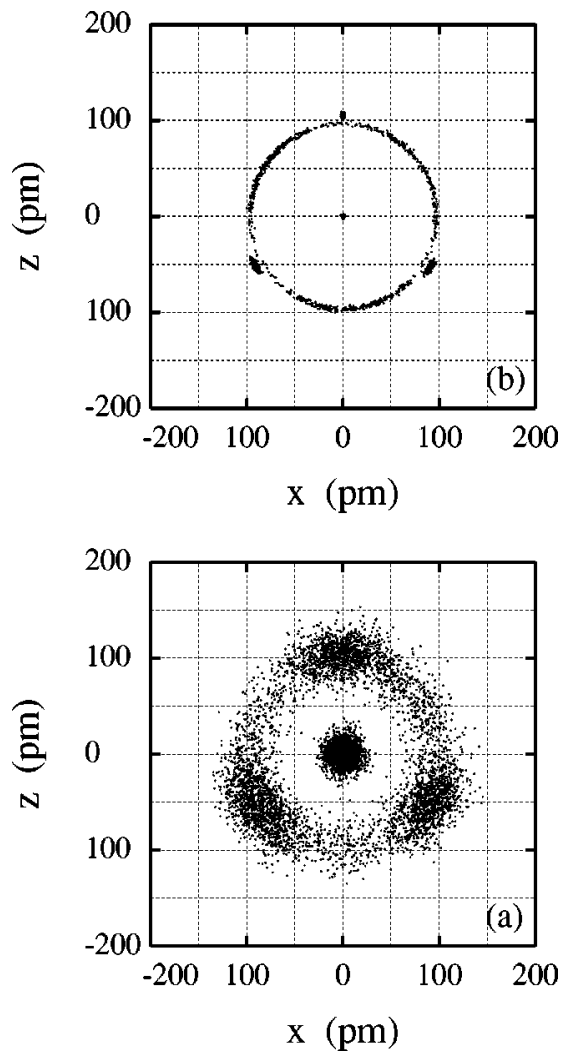

FIG. 4. Probability density function of the $\mathrm{C}_{2} \mathrm{H}_{5}$ nuclei projected onto the $x z$ plane as derived by quantum (a) and classical (b) simulations at $T$ $=25 \mathrm{~K}$.

ordinate $h$, which is defined in Fig. 5. $h$ measures the deviation of the radical fragment from the planar arrangement realized at $h=0$. The quantum and classical results for the pdf of the coordinate $h$ are presented in Fig. 6. The classical pdf is of bimodal form. The peaks are found close to the value of $h$ corresponding to the staggered minimum energy configuration. Remember that it is characterized by a pyramidal structure around the radical center. The bimodal distribution

TABLE IV. Canonical averages of bond lengths, $\langle d\rangle$, and some interatomic angles, $\langle\alpha\rangle$, of the ethyl radical as derived by classical and quantum MC simulations at $25 \mathrm{~K}$. The corresponding standard deviations $(\delta d, \delta \alpha)$ are also quoted. $\langle d\rangle$ and $\delta d$ are given in pm. The estimated error bars are lower than $0.1 \%$ for the mean values and lower than $0.5 \%$ for the standard deviations.

\begin{tabular}{|c|c|c|c|c|}
\hline \multirow[b]{2}{*}{ Bonds } & \multicolumn{2}{|c|}{ Classical MC } & \multicolumn{2}{|c|}{ PI MC } \\
\hline & $\langle d\rangle$ & $\delta d$ & $\langle d\rangle$ & $\delta d$ \\
\hline $\mathrm{C} 1-\mathrm{C} 2$ & 146.6 & 0.8 & 147.2 & 4.7 \\
\hline $\mathrm{C} 1-\mathrm{H} 1, \mathrm{C} 1-\mathrm{H} 2, \mathrm{C} 1-\mathrm{H} 3$ & 112.6 & 1.0 & 115.1 & 7.9 \\
\hline \multirow[t]{2}{*}{$\mathrm{C} 2-\mathrm{H} 4, \mathrm{C} 2-\mathrm{H} 5$} & 111.4 & 0.8 & 113.2 & 7.6 \\
\hline & \multicolumn{2}{|c|}{ Classical MC } & \multicolumn{2}{|c|}{ PI MC } \\
\hline Angles & $\langle\alpha\rangle$ & $\delta \alpha$ & $\langle\alpha\rangle$ & $\delta \alpha$ \\
\hline $\mathrm{C} 2-\mathrm{C} 1-\mathrm{H} 1$ & $111.6^{\circ}$ & $1.2^{\circ}$ & $111.5^{\circ}$ & $6.7^{\circ}$ \\
\hline $\mathrm{H} 2-\mathrm{C} 1-\mathrm{H} 3$ & $107.3^{\circ}$ & $1.8^{\circ}$ & $107.0^{\circ}$ & $8.0^{\circ}$ \\
\hline $\mathrm{H} 4-\mathrm{C} 2-\mathrm{H} 5$ & $116.4^{\circ}$ & $1.5^{\circ}$ & $116.4^{\circ}$ & $8.3^{\circ}$ \\
\hline $\mathrm{C} 1-\mathrm{C} 2-\mathrm{H} 4$ & $119.5^{\circ}$ & $1.5^{\circ}$ & $119.7^{\circ}$ & $7.0^{\circ}$ \\
\hline $\mathrm{C} 1-\mathrm{C} 2-\mathrm{b} 2$ & $160.1^{\circ}$ & $6.0^{\circ}$ & $161.7^{\circ}$ & $10.5^{\circ}$ \\
\hline
\end{tabular}




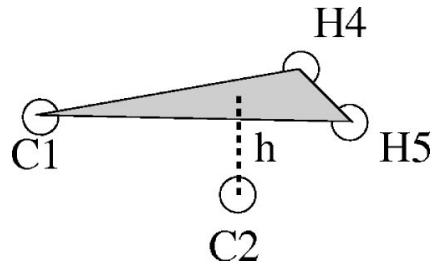

FIG. 5. Definition of the coordinate $h$ used to measure the planarity of the radical fragment in the ethyl radical. A planar configuration is characterized by $h=0$, while pyramidal configurations are characterized by $h \neq 0$.

is a consequence of the internal rotation of the $\mathrm{CH}_{2}$ group. The quantum pdf shows only one peak centered at $h=0$, i.e., the average structure of the $-\mathrm{CH}_{2}$ unit is planar. This result is a consequence of the anharmonicity of the zero-point motion of the atomic nuclei. It is in good agreement with the analysis of the high resolution spectrum of the $\mathrm{CH}_{2}$ rocking fundamental, which leads to the conclusion that the zero point and vibrationally excited states of ethyl are remarkably symmetrical. ${ }^{1}$

\section{B. Anharmonic effects in the vibrational wave numbers}

Anharmonic effects in the fundamental vibrational modes of ethyl are studied by applying the HLR approximation. This technique requires the calculation of the isothermal susceptibility tensor, $\chi^{T}$, defined in Eq. (2). The MC sampling of zero frequency modes in the form of molecular translations or global rotations leads to a singular susceptibility tensor. This has to be traced back to the fact that the eigenvalues of this tensor are inversely proportional to the mode wave number [see Eq. (3)]. A simple way to avoid this technical problem is to perform a MC sampling defined by collective random movements of the nuclei along the harmonic normal modes of the molecule. They are-by definition-orthogonal to the translational and rotational modes. Thus, a MCS in the PI MC runs for the calculation of the susceptibility tensor, $\chi^{T}$, implies: (i) an attempt of a sequential movement of each of the $N$ molecular "replicas"

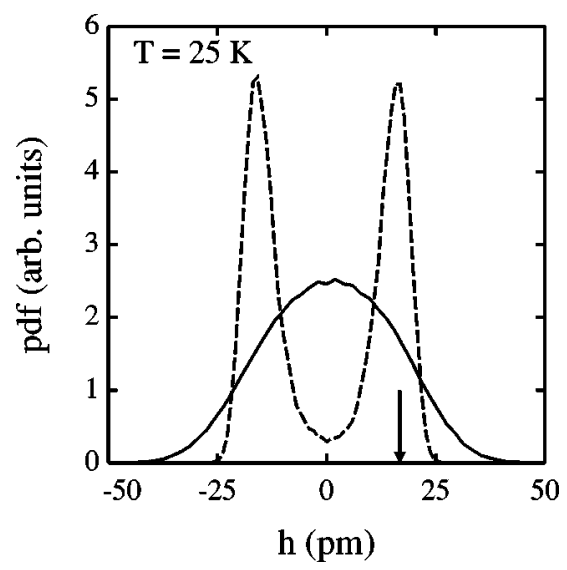

FIG. 6. Probability density function of the coordinate $h$ that characterizes the local configuration of the radical center in the ethyl radical. The continuous line is the result of the quantum PI MC simulation at $25 \mathrm{~K}$. The dashed line displays the classical MC result. The arrow points to the value of $h$ corresponding to the minimum energy configuration of the ethyl radical.
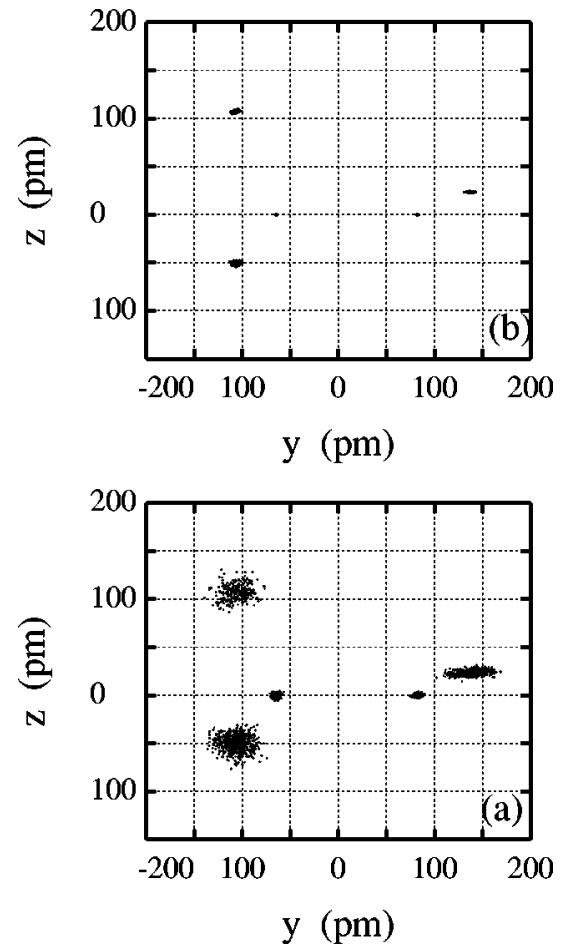

FIG. 7. (a) Probability density function of the $\mathrm{C}_{2} \mathrm{H}_{5}$ nuclei obtained by PI MC simulations at $25 \mathrm{~K}$ by sampling collective movements along selected vibrational modes. The MC sampling includes all vibrational modes except the rocking and the torsional modes. The displayed projection corresponds to the $y z$ plane. (b) Probability density function of the ring polymer centroids for the same simulation.

along each harmonic normal mode of the molecule; (ii) an attempt of a sequential movement of the centroids of the ring polymers along the harmonic normal modes of the molecule. This type of MC sampling has been applied satisfactorily to calculate the isothermal susceptibility tensor of molecules such as ethylene $e^{43}$ and $\mathrm{HOCl}^{46}$

The internal rotation of the ethyl radical causes an additional complication in the application of the HLR approximation. This dynamic process leads to the failure of the HLR approximation, for reasons analogous to its failure in the presence of translations or global rotations of the molecule. ${ }^{46}$ Test simulations have shown that the internal rotation of the molecule can be avoided by eliminating the rocking and torsional modes from the MC sampling. These modes are strongly coupled and act as active modes in the internal rotation, a conclusion which is in line with previous theoretical studies concerning vibrational effects on the torsional barrier of the ethyl radical. ${ }^{28}$ In Fig. 7(a), we display a $y z$ projection of the pdf of the $\mathrm{C}_{2} \mathrm{H}_{5}$ nuclei obtained with this type of sampling at $25 \mathrm{~K}$. This picture shows that the internal rotation is suppressed by this particular MC sampling. In Fig. 7(b), the pdf of the centroids, $X_{i}$, is displayed. From the recorded centroid configurations used to plot this figure, one can easily derive the covariances, $\delta X_{i} X_{j}$, of the centroid variables, that are required in the HLR approximation [see Eqs. (2) and (3)]. From this simulation, one gets information of all vibrational modes except the rocking and torsional ones. The anharmonicity of the rocking mode has been studied by additional simulations that include only the rocking 


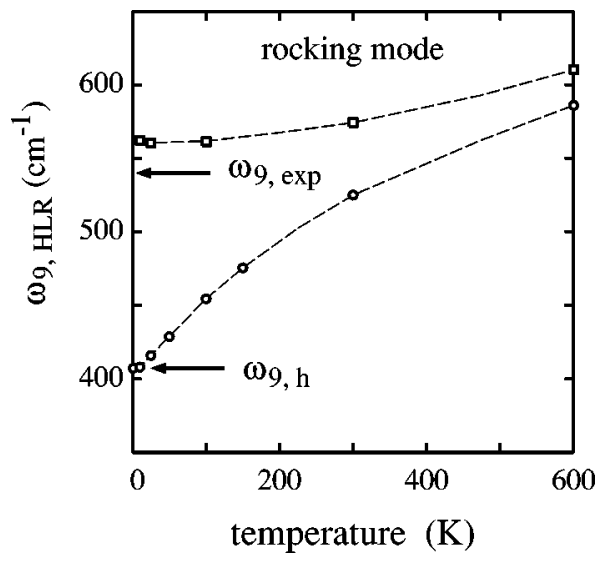

FIG. 8. Wave number of the fundamental rocking mode of the ethyl radical derived by the HLR approximation at different temperatures. The results obtained by quantum PI MC simulations are given by squares. The circles are results derived by classical MC simulations. $\omega_{9, h}$ is the wave number of the rocking mode in the harmonic approximation, and $\omega_{9 \text {,expt }}$ is the experimental wave number (Refs. 3 and 4).

mode in the MC sampling. As the torsional mode corresponds to an internal rotation it cannot be studied by the HLR approximation.

Before presenting the HLR results for the other vibrational modes, we display in Fig. 8 the results of the HLR approximation for the rocking mode at different temperatures. At the lowest studied temperatures, the wave number of the rocking mode, $\omega_{9, \mathrm{HLR}}=561 \mathrm{~cm}^{-1}$, is found to be larger than the harmonic result, $\omega_{9, h}$, by about $154 \mathrm{~cm}^{-1}$. The calculated anharmonic wave number is much closer to the experimental value of $540 \mathrm{~cm}^{-1}$ than the harmonic result. The wave numbers derived from classical MC simulations are also displayed in the figure. At low temperatures, the classical HLR approach correctly converges toward the harmonic result, $\omega_{9, h}$. However, at higher temperatures the HLR wave number increases due to the anharmonicity of the vibrational mode, approaching the results derived by the quantum simulations.

The anharmonic shifts in the wave numbers of the vibrational modes are defined as

$$
\Delta \omega_{i, \mathrm{HLR}}=\omega_{i, h}-\omega_{i, \mathrm{HLR}},
$$

where the $\omega_{i, h}$ are the harmonic wave numbers derived from the TB model (see Table III), and $\omega_{i, \mathrm{HLR}}$ are the wave numbers corresponding to the HLR approximation. The shifts, $\Delta \omega_{i, \mathrm{HLR}}$, obtained from our quantum simulations at $25 \mathrm{~K}$ are summarized in Table V. The torsional mode, $\omega_{15}$, has not been included in Table $\mathrm{V}$ as it corresponds to the internal rotation. The TB results are compared to the anharmonic shifts calculated as the difference between the harmonic wave numbers determined by ab initio methods $s^{15,17,18}$ and the experimental data of Pacansky et al., , $^{3,4}$

$$
\Delta \omega_{i}=\omega_{i, a b} \text { initio }-\omega_{i, \text { expt }} .
$$

From the three sets of $a b$ initio data, the TZ2Pf/B3LYP model ${ }^{18}$ leads systematically to the lowest anharmonic shifts, the MP2/6-31G* model $^{15}$ leads to the largest one. ${ }^{5}$ The results derived from the QCISD/6-3111G** data $^{17}$ lie in between. Our calculated shifts, $\Delta \omega_{i, \mathrm{HLR}}$, are positive for all
TABLE V. Anharmonic shifts in the vibrational wave numbers of the ethyl radical. The first column summarizes the results of the HLR approximation at $25 \mathrm{~K}$ by using the TB model. The other columns show the difference between the experimental wave numbers given by Pacansky et al. (Refs. 3 and 4) and the harmonic wave numbers corresponding to the $a b$ initio MP2/6-31G* calculation of Ref. 15, the QCISD/6-3111G** data of Ref. 17, and the TZ2Pf/B3LYP results of Ref. 18, respectively. The anharmonic shift corresponding to the stretching of the $\mathrm{C}-\mathrm{C}$ bond $\left(\Delta \omega_{7}\right)$ is given by considering two alternative assignments of the experimental wave numbers. All values are in $\mathrm{cm}^{-1}$.

\begin{tabular}{ccccc}
\hline \hline & TB & MP2 & QCISD & B3LYP \\
\hline$\Delta \omega_{1}$ & 278 & 211 & 132 & 109 \\
$\Delta \omega_{2}$ & 295 & 233 & 162 & 114 \\
$\Delta \omega_{3}$ & 291 & 222 & 191 & 101 \\
$\Delta \omega_{4}$ & 21 & & & \\
$\Delta \omega_{5}$ & 17 & & 59 & \\
$\Delta \omega_{6}$ & 16 & 105 & -61 & -78 \\
$\Delta \omega_{7}{ }^{\mathrm{a}}$ & 16 & -28 & 52 & 35 \\
$\Delta \omega_{7}{ }^{\mathrm{b}}$ & 16 & 85 & & -46 \\
$\Delta \omega_{8}$ & 24 & & -85 & 129 \\
$\Delta \omega_{9}$ & -154 & -75 & 156 & 91 \\
$\Delta \omega_{10}$ & 313 & 240 & 137 & 41 \\
$\Delta \omega_{11}$ & 305 & 212 & 68 & 19 \\
$\Delta \omega_{12}$ & 7 & 118 & 39 & \\
$\Delta \omega_{13}$ & 24 & 74 & & \\
$\Delta \omega_{14}$ & -21 & & & \\
\hline \hline
\end{tabular}

${ }^{a}$ Assignment $\omega_{7, \text { expt }}=1138 \mathrm{~cm}^{-1}$.

${ }^{\mathrm{b}}$ Assignment $\omega_{7, \mathrm{expt}}=1025 \mathrm{~cm}^{-1}$.

vibrational modes except for the rocking mode, $\omega_{9}$, and the mode $\omega_{14}$. Note that positive shifts imply a softening of the vibrational modes due to anharmonic corrections, while a negative shift is associated to a hardening of the mode. The largest positive anharmonic shifts correspond to the five vibrational modes $\left(\omega_{1}, \omega_{2}, \omega_{3}, \omega_{10}\right.$, and $\left.\omega_{11}\right)$ associated with the stretching of the $\mathrm{C}-\mathrm{H}$ bonds. This finding agrees with the anharmonic shifts, $\Delta \omega_{i}$, derived from the experimental and ab initio values. The negative anharmonic shift of the rocking mode derived in the TB model agrees in sign with the $a b$ initio based results.

Special interest deserves the analysis of the $\mathrm{C}-\mathrm{C}$ stretching mode, $\omega_{7}$. By using the accepted experimental assignment $^{3,4} \omega_{7, \text { expt }}=1138 \mathrm{~cm}^{-1}$, the anharmonic shift, $\Delta \omega_{7}^{a}$, of this vibrational mode results to be negative for the three sets of $a b$ initio data. However, the anharmonic shift for the $\mathrm{C}-\mathrm{C}$ stretch is predicted to be positive by the HLR approximation. This contradictory result leads us to suspect that the assignment of the band at $1138 \mathrm{~cm}^{-1}$ to the $\mathrm{C}-\mathrm{C}$ stretch mode is incorrect. In fact, anharmonic shifts of $\mathrm{C}-\mathrm{C}$ stretch vibrations of related molecules are systematically positive. For example, the values derived from Eq. (5) for $\mathrm{H}_{3} \mathrm{C}-\mathrm{CH}_{3}, \mathrm{H}_{2} \mathrm{C}-\mathrm{CH}_{2}$, and $\mathrm{HC}-\mathrm{CH}$ are all positive. They amount to 21,32 , and $63 \mathrm{~cm}^{-1}$, respectively. ${ }^{59}$ Considering that there are no other unassigned fundamentals in this region of the spectrum (see Table III), we suggest to assign this measured band-reported at $1138 \mathrm{~cm}^{-1}$ by Pacansky et al. ${ }^{3,4}$ and at $1133 \mathrm{~cm}^{-1}$ by Chettur et al. ${ }^{5}$-as overtone of the rocking fundamental at $540 \mathrm{~cm}^{-1}$. The rocking band is by far the strongest one in the IR spectrum of the ethyl radical. ${ }^{3-5}$ As a result of the negative harmonic shift found for this vibrational band, it seems reasonable to expect its 
TABLE VI. Corrections proposed for the assignment of experimental vibrational wave numbers of the ethyl radical. All values are in $\mathrm{cm}^{-1}$.

\begin{tabular}{ccc}
\hline \hline Expt. band & Previous assignment & New assignment \\
\hline $1138,{ }^{\mathrm{a}} 1133^{\mathrm{b}}$ & $\mathrm{C}-\mathrm{C}$ stretch & Overtone $\mathrm{CH}_{2}$ rocking \\
$1025^{\mathrm{b}}$ & $\mathrm{CH}_{3}$ rocking & $\mathrm{C}-\mathrm{C}$ stretch \\
\hline \hline
\end{tabular}

${ }^{\mathrm{a}}$ References 3 and 4.

${ }^{\mathrm{b}}$ Reference 5 .

overtone at a higher wave number $\left(1138 \mathrm{~cm}^{-1}\right)$ than twice the fundamental band $\left(1080 \mathrm{~cm}^{-1}\right)$. Moreover, a consistent explanation of the theoretical and experimental results can be achieved by the reassignment of the experimental vibrational band determined by Chettur et al. $^{5}$ at $1025 \mathrm{~cm}^{-1}$. We suggest that this peak is due to the $\mathrm{C}-\mathrm{C}$ stretch vibration (see Table III). We have presented the anharmonic shifts, $\Delta \omega_{7}^{b}$, in Table $\mathrm{V}$ as derived by this new assignment. Now they become positive in all cases. In Table VI, we summarize the proposed corrections in the assignment of the experimental vibrational bands. It should be mentioned that the assignment of the experimental peak at $1138 \mathrm{~cm}^{-1}$ to the $\mathrm{C}-\mathrm{C}$ stretch mode was considered by its authors as "by no means well established." 4 Unfortunately, this assignment has been currently accepted in the literature. ${ }^{3-5,18,22}$ Nevertheless, Hase et al. have pointed out the inconsistency of an experimental frequency that is larger than the $a b$ initio harmonic result for the $\mathrm{C}-\mathrm{C}$ stretch. $^{17}$

\section{Anharmonic effects in the internal energy}

The internal energy of the ethyl radical derived by PI MC simulations as a function of temperature is displayed in Fig. 9 by open squares. At the lowest temperature studied $(25$ $\mathrm{K})$, the internal energy amounts to approximately $1.5 \mathrm{eV}$. The results of the PI MC simulations are compared to the internal energy of two different sets of quantum harmonic oscillators.

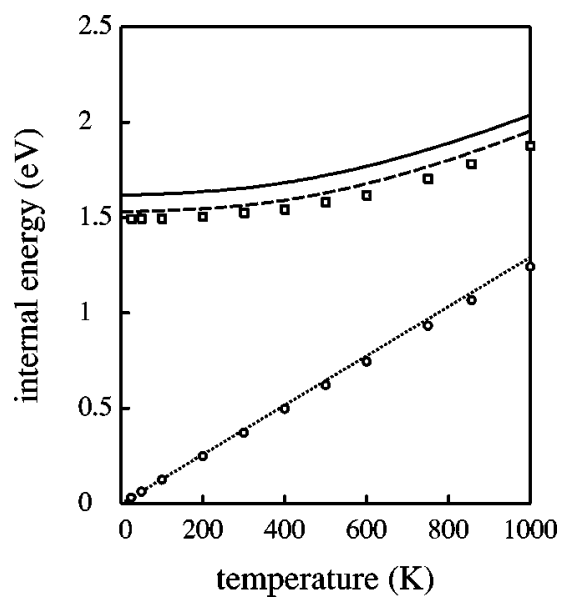

FIG. 9. Internal energy of the ethyl radical as a function of temperature. The results of the PI MC simulations are represented by squares. The continuous line shows the internal energy of a set of harmonic oscillators defined by the normal mode frequencies of the molecule. The dashed line shows the energy of a set of harmonic oscillators defined by frequencies determined by the HLR approximation. The results of classical MC simulations are given by open circles. while the classical limit in the harmonic approximation is represented by a dotted line.
The continuous line corresponds to the harmonic normal modes derived from the TB model, while the dashed line corresponds to oscillators defined by the HLR frequencies. In the latter case, we have used the harmonic result of $48 \mathrm{~cm}^{-1}$ for the torsional mode. It has been mentioned earlier that the HLR approach cannot be applied to study rotational modes. The anharmonic effect in the internal energy is measured by the deviation between the results corresponding to the harmonic normal modes and the PI MC approach. The harmonic model overestimates the internal energy by about $8 \%$ in the whole temperature range. However, a model defined by the HLR wave numbers improves the determination of the internal energy. Now we have a relative error of about $2 \%$ in the whole temperature range. This improvement demonstrates that the HLR approximation is able to account for anharmonic effects in the vibrational problem that are neglected in the harmonic approximation.

The comparison between kinetic and potential energy contributions to the internal energy provides further evidence of anharmonic effects in the ethyl radical. At $25 \mathrm{~K}$ the potential energy is found to be about $3 \%$ larger than the kinetic one, while at $1000 \mathrm{~K}$ the kinetic energy is about $3 \%$ larger than the potential one. Note that both the kinetic and potential energy should be equal in the harmonic approximation.

The results of classical MC simulations for the internal energy in Fig. 9 are shown as open circles. The dotted line represents the classical limit in the harmonic approximation. The deviation of the simulation results from the harmonic approximation is noticeable only at the highest temperatures studied, e.g., at $1000 \mathrm{~K}$ both results differ by about 4\%. This anharmonic behavior contrasts to the quantum case. Here the anharmonic correction at low temperatures, caused by the zero-point motion of the nuclei, is found to be comparable to the anharmonic effect at the highest temperature studied.

\section{Possibility of coherent tunneling}

The ESR spectra of the ethyl radical trapped in a solid ethane matrix change drastically when the temperature decreases below $30 \mathrm{~K} .{ }^{9}$ A definite explanation of this effect is still missing. In this work we want to check whether a coherent tunneling process involving the atom $\mathrm{H} 1$ in the staggered structure of ethyl might be responsible for this behavior. The tunneling process corresponds to the jump of the H1 atom between the methyl and methylene groups (see Fig. 1). A prerequisite for this tunneling process is the suppression of the internal rotation of the $\mathrm{CH}_{2}$ group. This behavior renders possible so that the $\mathrm{H} 1$ atom can resonate between two symmetry equivalent positions. The high degree of crystalline order in the ethane matrix used in the experimental setup might be a factor favoring this condition.

A fingerprint for coherent tunneling in a PI MC simulation is the spreading of the atomic nuclei over the two symmetry equivalent positions involved in the tunneling process. ${ }^{60,61}$ Therefore, we have performed simulations at 25 $\mathrm{K}$ where the ring polymer of the $\mathrm{H} 1$ atom initially spreads over the two symmetry equivalent positions. This tunneling configuration of the ethyl radical is displayed in Fig. 10. The main result of these simulations is that the tunneling configu- 


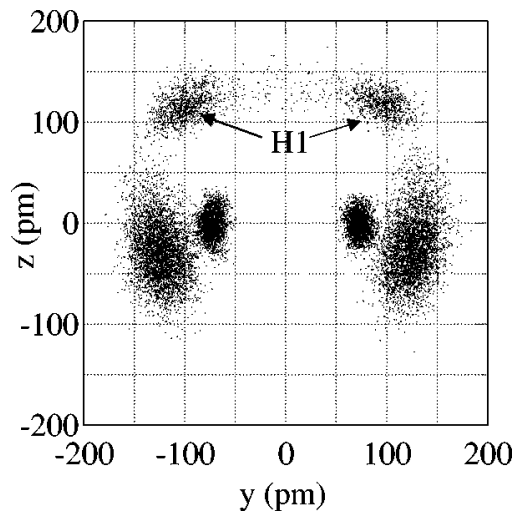

FIG. 10. Probability density function of the $\mathrm{C}_{2} \mathrm{H}_{5}$ nuclei for a configuration where the $\mathrm{H} 1$ atom tunnels between two symmetry equivalent positions. The projection is displayed in the $z y$ plane. The picture corresponds to an equilibration run at $T=25 \mathrm{~K}$.

ration appears as a metastable structure under the employed numerical conditions. During the equilibration run, it jumps spontaneously to the staggered ethyl structure. This behavior is illustrated in Fig. 11 by the monitorization of the kinetic energy along the simulation. The tunneling configuration is characterized by a lower kinetic energy. This fact is a consequence of the Heisenberg uncertainty principle. The larger spatial uncertainty of the atomic nuclei in the tunneling configuration is associated with a lower momentum uncertainty, that translates into a lower kinetic energy. Along the simulation run, the initial tunneling structure reverts toward a normal staggered structure with higher kinetic energy. Therefore, the PI MC simulations do not provide any evidence for a coherent tunneling process of a hydrogen atom between the $\mathrm{CH}_{3}$ and $\mathrm{CH}_{2}$ groups at low temperatures.

\section{CONCLUSIONS}

We have presented full quantum simulations of the ethyl radical. For this purpose we have combined a tight-binding model to describe the potential energy surface of the electronic ground state, with path integral Monte Carlo simula-

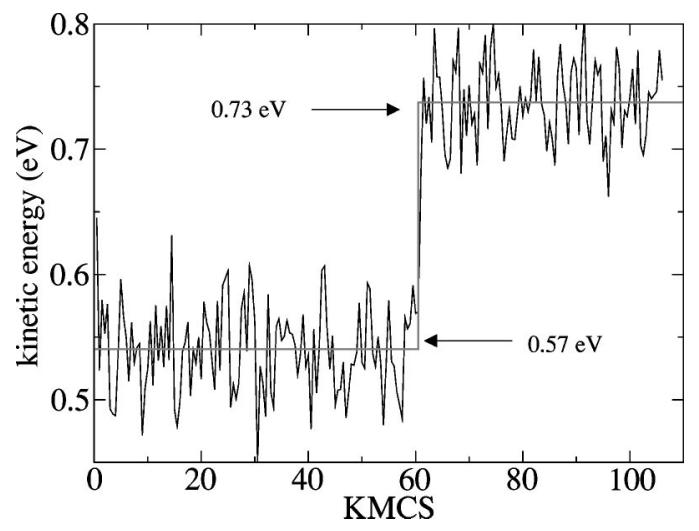

FIG. 11. Result of the monitorization of the kinetic energy along the equilibration run of an initial tunneling structure at $25 \mathrm{~K}$. The tunneling structure is characterized by a kinetic energy around $0.57 \mathrm{eV}$. This structure changes during the equilibration run to a staggered structure characterized by a higher kinetic energy. A KMCS is defined as $10^{3}$ MCS. tions to describe the quantum nature of the atomic nuclei at finite temperatures. The tight-binding model has been compared to available $a b$ initio calculations based on both correlated approaches on the basis of the Hartree-Fock determinant and density functional theories. We have shown that the tight-binding model provides a reasonable description of the geometry and relative energies of the staggered and eclipsed configurations of the ethyl radical, and also of its harmonic vibrational wave numbers.

The quantum simulations have allowed us to quantify the influence of the anharmonicity of the interatomic potential on some equilibrium properties of this molecule. An interesting anharmonic result is the planar structure of the radical fragment. Thus, the averaged zero-point structure is more symmetrical than the minimum energy configuration, characterized by a nonplanar $-\mathrm{CH}_{2}$ unit. The hydrogen atoms of the methyl group become dynamically equivalent as a consequence of the internal rotation of the molecule. In the vibrational ground state, we find an increase of $0.4 \%$ in the equilibrium $\mathrm{C}-\mathrm{C}$ bond length due to anharmonic effects, while the increase is about $2 \%$ for $\mathrm{C}-\mathrm{H}$ bond lengths. The standard deviation of the $\mathrm{C}-\mathrm{C}$ bond length distribution amounts to about $3 \%$ of the average bond distance. For the $\mathrm{C}-\mathrm{H}$ bonds, we predict larger quantum fluctuations due to zero-point effects. They amount to roughly $7 \%$ of the corresponding mean value. The anharmonic contribution to the internal energy amounts to about $8 \%$ in the studied temperature range $25-1000 \mathrm{~K}$.

Interesting anharmonic effects are found in the vibrational modes of the ethyl radical. A nonperturbative approach based on the relation between the centroid density and linear response theory has been applied to calculate anharmonic effects in the vibrational wave numbers. The well-known anharmonic corrections, i.e., the softening of the mode, are found for all vibrational modes except two. The most important inverse anharmonic correction, i.e., a hardening of the mode, occurs for the rocking mode. This result agrees with anharmonic shifts that are expected by comparison of experimental and harmonic ab initio results for this mode. We find that the anharmonicity in the $\mathrm{C}-\mathrm{C}$ stretching mode leads to a softening of this mode. This result bends us to propose a new assignment of the experimental vibrational band associated with the $\mathrm{C}-\mathrm{C}$ stretching mode. The previously accepted assignment demands an inverse anharmonic effect that is inconsistent with our anharmonic results and also with the anharmonic behavior found in related molecules.

We have explored the possibility for coherent tunneling of a hydrogen atom between the methyl $\left(\mathrm{CH}_{3}\right)$ and methylene $\left(\mathrm{CH}_{2}\right)$ groups of the ethyl radical at temperatures below $30 \mathrm{~K}$. The results of the quantum simulation suggest that the probability for this tunneling process is extremely small. Therefore, this mechanism cannot explain the change in the ESR spectrum of the ethyl radical in an ethane matrix below $30 \mathrm{~K}$.

\section{ACKNOWLEDGMENT}

The present work has been supported by CICYT (Spain) through Grant No. BFM2000-1318. 
${ }^{1}$ T. J. Sears, P. M. Johnson, P. Jin, and S. Oatis, J. Chem. Phys. 104, 781 (1996).

${ }^{2}$ T. Kumada, J. Kumagai, and T. Miyazaki, J. Chem. Phys. 114, 10024 (2001).

${ }^{3}$ J. Pacansky and J. Dupuis, J. Am. Chem. Soc. 104, 415 (1982).

${ }^{4}$ J. Pacanski and B. Schrader, J. Chem. Phys. 78, 1033 (1983).

${ }^{5}$ G. Chettur and A. Snelson, J. Phys. Chem. 91, 3483 (1987).

${ }^{6}$ N. Sogoshi, T. Wakabayashi, T. Momose, and T. Shida, J. Phys. Chem. A 101, 522 (1997).

${ }^{7}$ T. J. Sears, P. M. Johnson, and J. BeeBe-Wang, J. Chem. Phys. 111, 9213 (1999).

${ }^{8}$ S. Davis, D. Uy, and D. J. Nesbitt, J. Chem. Phys. 112, 1823 (2000).

${ }^{9}$ F. J. Adrian, J. Bohandy, and B. F. Kim, J. Chem. Phys. 100, 8010 (1994).

${ }^{10}$ P. W. Percival, J. Brodovitch, S. Leung et al., Chem. Phys. Lett. 163, 241 (1989).

${ }^{11}$ D. G. Fleming, J. J. Pan, M. Senba, D. J. Arseneau, R. F. Kiefe, M. Y. Shelley, S. F. J. Cox, P. W. Percival, and J. Brodovitch, J. Chem. Phys. 105, 7517 (1996).

${ }^{12}$ K. Toriyama, M. Iwasaki, K. Nunome, and H. Muto, J. Chem. Phys. 75, 1633 (1981).

${ }^{13}$ C. A. McDowell, P. Raghunathan, and K. Shimokoshi, J. Chem. Phys. 58, 114 (1973).

${ }^{14}$ F. J. Adrian, E. L. Cochran, and V. A. Bowers, J. Chem. Phys. 59, 3946 (1973).

${ }^{15}$ B. Ruscic, J. Berkowitz, L. A. Curtiss, and J. Pople, J. Chem. Phys. 91, 114 (1989).

${ }^{16}$ J. Dyke, A. Ellis, and A. Morris, J. Phys. Chem. 88, 2565 (1984).

${ }^{17}$ W. L. Hase, H. B. Schlegel, V. Balbyshev, and M. Page, J. Phys. Chem. 100, 5354 (1996).

${ }^{18}$ I. S. Ignatyev, Y. Xie, W. D. Allen, and H. F. Schaefer III, J. Chem. Phys. 107, 141 (1997).

${ }^{19}$ J. C. Rienstra-Kiracofe, W. D. Allen, and H. F. Schaefer III, J. Phys. Chem. A 104, 9823 (2000).

${ }^{20}$ A. Zyubin, A. Mebel, and S. Lin, Chem. Phys. Lett. 323, 441 (2000).

${ }^{21}$ J. Villà, A. González-Lafont, J. M. Lluch, and D. G. Truhlar, J. Am. Chem. Soc. 120, 5559 (1998).

${ }^{22}$ Y. Chen, A. Rauk, and E. Tschuikow-Roux, J. Chem. Phys. 93, 1187 (1990).

${ }^{23}$ I. Carmichael, J. Phys. Chem. 95, 6198 (1991).

${ }^{24}$ D. M. Chipman, J. Chem. Phys. 94, 6632 (1991).

${ }^{25}$ H. Suter and T.-K. Ha, Chem. Phys. 154, 227 (1991).

${ }^{26}$ D. Buttar and B. C. Webster, J. Chem. Soc., Faraday Trans. 87, 2901 (1991).

${ }^{27}$ B. Webster and D. Buttar, J. Chem. Soc., Faraday Trans. 92, 2331 (1996).

${ }^{28}$ P. M. Johnson and T. J. Sears, J. Chem. Phys. 111, 9222 (1999).

${ }^{29}$ A. L. East and P. Bunker, Chem. Phys. Lett. 282, 49 (1998).
${ }^{30}$ J. A. Seetula, J. Chem. Soc., Faraday Trans. 94, 1933 (1998).

${ }^{31}$ R. P. Feynman and A. Hibbs, Quantum Mechanics and Path Integrals (McGraw-Hill, New York, 1965).

${ }^{32}$ H. Kleinert, Path Integrals (World Scientific, Singapore, 1995).

${ }^{33}$ D. Porezag, T. Frauenheim, T. Köhler, G. Seifert, and R. Kaschner, Phys. Rev. B 51, 12947 (1995).

${ }^{34}$ J. Widany, T. Frauenheim, T. Köhler, M. Sternberg, D. Porezag, G. Jungnickel, and G. Seifert, Phys. Rev. B 53, 4443 (1996).

${ }^{35}$ H.-P. Cheng, R. Barnett, and U. Landman, Chem. Phys. Lett. 237, 161 (1995).

${ }^{36}$ M. Valladares, A. Fisher, and W. Hayes, Chem. Phys. Lett. 242, 1 (1995).

${ }^{37}$ D. Marx, M. E. Tuckerman, J. Hutter, and M. Parrinello, Nature (London) 397, 601 (1999).

${ }^{38}$ M. Benoit, D. Marx, and M. Parrinello, Nature (London) 392, 258 (1998).

${ }^{39}$ D. Marx and M. Parrinello, Science 284, 59 (1999).

${ }^{40}$ R. O. Weht, J. Kohanoff, D. A. Estrin, and C. Chakravarty, J. Chem. Phys. 108, 8848 (1998).

${ }^{41}$ M. E. Tuckerman and D. Marx, Phys. Rev. Lett. 86, 4946 (2001).

${ }^{42}$ R. Ramírez, E. Hernández, J. Schulte, and M. Böhm, Chem. Phys. Lett. 291, 44 (1998).

${ }^{43}$ R. Ramírez, J. Schulte, and M. Böhm, Mol. Phys. 99, 1249 (2001).

${ }^{44}$ M. Böhm, J. Schulte, and R. Ramírez, Chem. Phys. Lett. 332, 117 (2000).

${ }^{45}$ M. Böhm, J. Schulte, and R. Ramírez, Int. J. Quantum Chem. 86, 280 (2002).

${ }^{46}$ R. Ramírez and T. López-Ciudad, J. Chem. Phys. 115, 103 (2001).

${ }^{47}$ C. M. Goringe, D. R. Bowler, and E. Hernández, Rep. Prog. Phys. 60, 1447 (1997).

${ }^{48}$ R. P. Feynman, Statistical Mechanics (Addison-Wesley, New York, 1972).

${ }^{49}$ M. J. Gillan, in Computer Modeling of Fluids, Polymers, and Solids, edited by C. R. A. Catlow, S. C. Parker, and M. P. Allen (Kluwer, Dordrecht, 1990).

${ }^{50}$ D. M. Ceperley, Rev. Mod. Phys. 67, 279 (1995).

${ }^{51}$ C. Chakravarty, Int. Rev. Phys. Chem. 16, 421 (1997).

${ }^{52}$ N. Metropolis, A. Rosenbluth, M. Rosenbluth, A. Teller, and E. Teller, J. Chem. Phys. 21, 1087 (1953).

${ }^{53}$ J. Cao and B. J. Berne, J. Chem. Phys. 91, 6359 (1989).

${ }^{54}$ M. Böhm, J. Schulte, E. Hernández, and R. Ramírez, Chem. Phys. 264, 371 (2001).

${ }^{55}$ J. Cao and G. A. Voth, J. Chem. Phys. 100, 5093 (1994).

${ }^{56}$ J. Cao and G. A. Voth, J. Chem. Phys. 100, 5106 (1994).

${ }^{57}$ J. Cao and G. A. Voth, J. Chem. Phys. 101, 6157 (1994).

${ }^{58}$ R. Ramírez and T. López-Ciudad, J. Chem. Phys. 111, 3339 (1999).

${ }^{59}$ B. G. Johnson, P. M. Gill, and J. A. Pople, J. Chem. Phys. 98, 5612 (1993).

${ }^{60}$ J. C. Noya, C. P. Herrero, and R. Ramírez, Phys. Rev. Lett. 79, 111 (1997).

${ }^{61}$ J. C. Noya, C. P. Herrero, and R. Ramírez, Phys. Rev. B 56, 15139 (1997). 